\begin{tabular}{|c|c|}
\hline & Volume \& Issues Obtainable at The Women University Multan \\
\end{tabular}

\title{
Mediating Role of Rumination between Neuroticism and Depression among the Married and Unmarried Nurses of Multan
}

\author{
Syed Muzaffar Hussain ${ }^{1}$, Saralah Devi Mariamdaram Chethiyar ${ }^{2}$, Nabisah Binti \\ Ibrahim $^{3}$
}

\footnotetext{
${ }^{1}$ Psychology \& Counselling Program, School of Applied Psychology, SocialWork and Policy, College of Arts and Sciences, Universiti Utara Malaysia

${ }^{2}$ Psychology\& Counselling Program, School of Applied Psychology, SocialWork and Policy, College of Arts and Sciences, Universiti Utara Malaysia

${ }^{3}$ Psychology\& Counselling Program, School of Applied Psychology, SocialWork and Policy, College of Arts and Sciences, Universiti Utara Malaysia
}

\begin{tabular}{lc}
\hline ARTICLE DETAILS \\
\hline History: & October 31,2021 \\
Received: & November 16, 2021 \\
Review: & December 16, 2021 \\
Accepted: & December 20, 2021
\end{tabular}

\section{Keywords: \\ Rumination \\ Neuroticism \\ Depression}

Nurses

Multan

\section{DOI:}

10.52700/assap.v2i2.100

\begin{abstract}
The present research was directed to study the mediating role of rumination between neuroticism and depression among the married and unmarried nurses of Multan. Data was taken from $(n=150)$ nurses through a stratified proportional sampling method. Hamilton Depression Rating Scale, Ruminative Response Scale and Big Five Inventory were utilized as an assessment measure in the present research. SPSS-21 version was used in the current research. Pearson product-moment correlation analysis revealed that rumination and neuroticism have a significant positive $(P=.05)$ relationship with depression. However, hierarchical regression analysis by Baron and Kenny method shows that rumination shows a significant mediating $(P=.05)$ role between neuroticism and depression. Consequences determined that a higher level of ruminating thoughts and neuroticism would lead to depression. The present study will help to understand the consequences of depression, especially among nurses. Existing policies and coping strategies could be inquired and could probably to improve to better help this population. Based on this study, it is not only particular for professionals or nurses to be aware of depression toward a better life in the Pakistani society but also to be educated on the culture itself and clinical area.

(C) 2021 The Authors, Published by WUM. This is an Open Access Article under the Creative Common Attribution Non Commercial 4.0
\end{abstract}

Corresponding author's email address: syedbzu@gmail.com 


\section{Introduction}

Depression is one of the utmost general health issues, presently affecting nurses. This illness harms a person's personal and professional life (Ibrahim et al. 2013). Depression is frequently an enduring and repetitive process that seriously impairs the life of the nurses and even their families, and causes high levels of daily impairments (Nutt et al. 2007). The important characteristics of depressive diseases are constant low mood and loss of interest or pleasure in usually pleasant activities (Allan \& Ebmeier, 2013). However, depression is identified as one of the utmost expensive and typical diseases worldwide, and its huge financial load is derived from its high occurrence and considerable functional disabilities involved in the disease (WHO, 2008).

Therefore, the current study aimed to examine the mediating role of rumination between neuroticism and depression among the married and unmarried nurses of Multan. The current study was led to support mental health professionals and Pakistani people particularly nurses to understand how much rumination and neuroticism show a significant effect in depression. The basic aim of the present research is to provide insight into both clinical and social situations and create an awareness that how policymakers, community workers, and mental health professionals reduce depression and improve the lives of nurses in a better way.

\subsection{Rumination}

Rumination mentions to as recurrent and passive self-focused ideas revolving around negative emotions and depression of a person (Hoeksema, 1991). Evidence' indicating continuously from the last three decades that rumination is a significant predictor of depression (Fang et al. 2019). Support for the response styles theory has been firm in the adult researches mostly the association between rumination and succeeding growths in depressive signs and the start of major depression (Moult et al. 2019). Researchers describe that depressive rumination has strongly linked with increasing, maintenance ( $\mathrm{Li}$ et al. 2017) and seem to support factors to the onset of depression. Now researchers are convinced that rumination has become a cognitive risk aspect for the onset of depression and relapse (Wang et al. 2015). Many researchers are noticing in their studies that rumination progresses not only in depression patients but also in those who have had past experiences of depression (Jacob et al. 2020). The researchers explain rumination has emerged in the etiology and management of major depression. Most of the time it also exhibits increased levels of rumination and an exaggerated focus on negative events and repetitive thinking patterns with MDD (Jacob et al. 2020). Nevertheless, it has been seen, this type of situation occurs to nurses when they attend the patients and during the duty timings.

Moreover, rumination increases the pessimistic attitude by increasing the severity and proportion of memorizing negative events. Rumination through focusing on the standards that the self has not achieved and it might direct to negative affect. Emphasizing positive accomplishments is not associated with negative affect conditions, such as depressive illnesses (Broderick \& Korteland, 2004). Depressive rumination is recurrent devotion to negative attitudes regarding one's self and is significantly related to depressive illnesses. A substantial number of studies have revealed that rumination preserves negative effects, anticipates depressive eras and eventuates the long-lasting depressive periods (Lopez et al. 2016). 
In current years, new philosophies have focused that the prior mood affects the ideas, thoughts and attitudes. In these approaches, a negative mood is direct to rumination (Yousefi et al. 2008). Our outcomes are constant with Beck's cognitive theory of depression and response styled theory's extension of Beck's theory of rumination in response to negative events. Two anticipated approaches that reveal emotional vulnerabilities to depression are the cognitive responses of threatening and rumination. Trait affective susceptibilities have been revealed to predict depressive signs by ruminative cognitive responses to optimistic and negative life events. Trait negative affect predicts high levels of rumination on negative conditions and high depressive signs. The effective changes of high negative affect might predict depressive symptoms by harming the effect of stressors on following negative mood through cognitive reactions such as ruminating in response to stressful events, which, over time can lead to depressive signs (Neuendorf et al. 2016). The results can be interpreted in this process: when people experience positive effects, maybe they don't involve in negative emotions and thoughts. Hereafter, they are less likely to experience negative emotional consequences such as depression.

\subsection{Neuroticism}

Neuroticism is one of the Big Five higher-order personality traits in the research of psychology. The outcomes from the present study showed Neuroticism is a constant and genetic personality trait, Individuals whose high score are more likely comparatively average to be moody and is strongly linked to depression ( $\mathrm{Li}$ et al. 2019) and has direct relations to future signs of depression and as well as sometimes significant indirect relations to future levels of depression (Sørengaard et al. 2019). Previous results of many studies pointed out, particularly neuroticism plays a significant role in the expansion of depression (Bienvenu et al. 2003) and significantly predict depression (Clark et al. 1994). However, to the best of our knowledge, neuroticism plays a role positively in association with $22 \%$ of the relation to depression. A considered number of epidemiological studies reported depression and neuroticism (a personality trait) are associated with each other and both are common comorbidities (Muris et al. 2004).

Furthermore, neuroticism is associated with both stress and depression. Several longitudinal pieces of research have revealed neuroticism to be a predictor of both consequent diagnoses and chronicity of major depression (Murray \& Nill, 2019). Furthermore, neuroticism is cross-sectionally and longitudinally related to episodic life stress (Meng et al. 2020).

Some studies have examined the relations among neuroticism, depression, and life stress simultaneously. Researchers revealed those with high neuroticism were more likely to experience a depressive episode following stressful life events than those with low neuroticism were. (Klein et al. 2009) revealed that neuroticism moderately mediated the potential relationship between long-term problems (a form of CLS) and psychological distress (signs of dysphoria, sadness, and nervousness).

\subsection{Depression}

Depression (major depressive disease) is a usual and severe psychological disorder that negatively influences how you feel, the method you deliberate and in what way you behave. Depression generates feelings of unhappiness and/or a diminish of interest in activities you once liked. It can direct to a diversity of psychological and physiological issues and can 
reduce your skill to function at work and home. Depression is a mood disease that avoids persons from directing a standard life, at work publicly or within their family. Depression is a general psychological disorder, depicting a low mood, low self-esteem and self-worth, feel guilty. Depression leads to poor sleep and loss of appetite, affecting people's thinking, behavior and physical health (Fanous et al. 2002).

Major depressive disorder (MDD) is an extremely prevailing, etiologically multifactorial and clinically assorted illness. It is the most significant psychological illness concerning public health effects. Depressive illnesses are classified as the fourth important reason of issue among all illnesses. If recent inclinations for covariates and epidemiological change endure, the problem of depression will rise and through the year 2020 depression will become another main reason for disability-adjusted life years vanishing. International will be ischemic heart illness and in the advanced counties, depression will be the top-ranking reason of illness (Ormel \& Wohlfarth, 1991). Additionally, Depression can disturb persons at every stage of their lifetime. It is fundamentally an occasional periodic illness. Epidemiological researches indicate that intervention for depression is frequently poor or is unknown. Depression seems to be a prolonged disease with a higher risk of relapse above one's lifetime, particularly when satisfactory management is not accessible. Considerable amounts of depressive clients end up their lives by committing suicide. Suicide is the utmost general consequence of depression (APA, 2013).

Depression is not only a typical, frequently enduring, and persistent illness, but it is cardinally related to a substantial loss in occupational and daily societal and mental healthiness. Major depressive disorder (MDD) is the fourth huge cause for disability globally and is predicted to become the second basic reason by the year 2020. The results of medical research (Murray \& Lopez, 1997) revealed that depressed people have similar or severe physiological, psychosocial, and role functioning comparatively those who have long-lasting medical conditions. Similarly, the World Health Organization (WHO) collective research on mental issues in overall healthcare (Sokero et al. 2003) described high working disability, even after inhibiting for physiological illnesses severity among patients with depression. Moreover, extensively a client consistently symptomatic, the lower the probabilities of a comprehensive recovery and therefore higher dysfunction.

\subsection{Rationale/Objective}

The current research aimed to examine the mediating role of rumination between neuroticism and depression among the married and unmarried nurses of Multan. The present study was conducted to fulfill the previous gap. The current study was led to support mental health professionals and Pakistani people particularly nurses to understand how much rumination and neuroticism show a significant effect in depression. The basic aim of the present research is to provide insight into both clinical and social situations and create an awareness that how policymakers, community workers and mental health professionals reduce depression and improve the lives of nurses in a better way.

\section{Methodology}

\subsection{Participants}

It is correlational research directed with nurses of Multan. A correlational research design was used. We recruited $(n=150)$ nurses through a stratified proportional sampling method in 
Multan, Pakistan. The sample consisted of 150 nurses in which 120 (80\% of the sample unmarried), 20 (13.2\% of the sample married), and 10 (6.6\% of sample single/separated). The research was approved by the Human Research Ethics Committee at the Children Hospital Multan.

\subsection{Measures}

\subsubsection{Demographic Questionnaires}

A set of queries were established to capture appropriate personal information regarding nurses. Nurses' details included age, marital status and number of children were the demographic variables of the current research.

\subsubsection{Ruminative Responses Scale (RRS; Hoeksema, 1994)}

The Ruminative Response Scale (TSS) was originally established by (Hoeksema, 1994) to measure the level of rumination. The scale contains 22 items with a 4-point Likert scale (1= rarely, $4=$ almost always). The tool has three dimensions a) brooding b) reflection and c) depression-related. The score ranges of the scale were from minimum 22 to maximum 88 and high scores indicate high rumination and low scores indicate low rumination. However, rumination has not been clearly distinguished from depression. Some of the 22 items on the RRS overlap with depressive symptomology (34). The Cronbach's alpha reliability of this scale is $(\alpha=.88$ to .99$)(34)$.

\subsubsection{Big Five Personality Inventory (BFI; McCrae \& John, 1992)}

Big Five Personality Inventory was originally developed (McCrae \& John, 1992). Big Five, Inventory is a measurement scale that encompasses five basic dimensions of personality. Neuroticism is one of five of them is among the most comprehensive and popular methods to determine personality traits (35) and indicate as a psychological trait and higher scores among females, (36). BFI is an inventory consisting of 44 questions that assess the Big Five Factors of personality (dimensions) (37). The Big Five Factors are recreated from John and Srivastava in 1999 and neuroticism is one of them (38). Neuroticism (the inverse of emotional stability was measured using the Big-Five inventory. Participants indicated on a Likert Scale ranging from with on the scale 1-5, where $1=$ disagree, $2=$ slightly disagree, $3=$ neutral, $4=$ slightly agree and $5=$ agree $(38,39,40,41)$. The Cronbach's alpha reliability of this scale is $(\alpha=.83)$.

\subsubsection{Hamilton Depression Rating Scale (HDRS; Hamilton, 1960)}

Hamilton Depression Rating Scale was originally developed by (Hamilton, 1960). Also measures signs of the previous week and involves nine questions rated 0 to 4 and eight items rated 0 to 2, for a score range of $0-52$ (42). HDRS is used to rate the severity of depression through assessing mood, insomnia, feelings of guilt, anxiety, suicide ideation, weight loss, retardation or agitation, and somatic symptoms. Participants with a score of 0 to 7 points were considered normal, 8 - 13 as mild depression, 14 - 18 as moderate depression, 19 - 22 as severe depression and $>23$ as very severe depression (Hamilton, 1960). The Cronbach's alpha reliability of this scale is $(\alpha=.95)$. 


\subsection{Procedure}

This study was accepted by the Institutional Review Board of the Psychology and Counselling Program, School of Applied Psychology, SocialWork and Policy, College of Arts and Sciences, Universiti Utara Malaysia. Before data collection could start, consent to select participants was required from school administrators and once consent was taken nurses were approached. Nurses were systematically informed regarding the purpose of the study and those who had permission were given the form to anonymously complete. Meanwhile, this research employed paper-and-pencil scales for data collection. Those who agreed to participate in the research were given a set of forms to fill. Questionnaires that were completed were concurrently ready for data analyses.

\subsection{Analysis}

Statistical analyses were conducted using the IBM SPSS Statistics: version 22 with significance set at $(\mathrm{P}=.05)$. The demographic variables showed participants were drawn from the ongoing working hospital and nursing hostel, who were aged 21-30 (78.5\%) $=120, \quad 31$ $40(16.6 \%)=25$ and $41-50(4 \%)=6$ at the initial assessment. The participants were belonging to city $98(64.9 \%)$, town $29(17.2 \%)$ and village $27(17.9 \%)$. The sex split was $151(100 \%)$ females. For the main study variables to ensure easy analysis and understanding, Pearson product-moment correlation analysis, hierarchal regression analysis and mediation analysis were used.

\section{Results}

\subsection{Pearson Product Moment Correlation Analysis}

The outcomes of the correlation analyses are reported in Table 3. A significant positive relationship was observed between the total score of rumination $(r=.80, P=.05)$ with depression and neuroticism has also significant negative relationship $(r=.42, P=.05)$ with depression. See Table 2 for the summary.

\subsection{Hierarchal Regression Analysis}

Hierarchical regression was shown with rumination entered at the first step and neuroticism entered at the second step. The results for the regression analysis are in $\underline{3}$. Findings revealed that both models were important. In the first step $\left(\Delta R^{2}=.11, F(1,147)=.20, P=.00\right)$ rumination was a statistically significant predictor $(\beta=.80, P=.00)$, recommending that rumination predicted depression. In the second model $\left(\Delta R^{2}=.31, F(2,146)=17.8, P=.01\right)$ neuroticism $(\beta=.42, P=.01)$ was significant predictor of depression. In sum, the results revealed that rumination and neuroticism were significant predictors of depression.

\subsection{Mediation Analysis}

Results of direct effect revealed that neuroticism and rumination were significant positive $(P=.05)$ predictors of depression. The result of an indirect effect of rumination revealed that rumination was a significant $(P=.05)$ mediator between neuroticism and depression. 
Table 1: Psychometric Properties of Questionnaires $(n=150)$

\begin{tabular}{lllll}
\hline Variables & $\boldsymbol{K}$ & M & SD & $\boldsymbol{\alpha}$ \\
\hline Rumination Ruminative Scale & 22 & 38.71 & 27.0 & .99 \\
\hline Big Five Personality Inventory & 44 & 23.52 & 11.2 & .83 \\
\hline Hamilton Depression Rating Scale & 09 & 60.40 & 16.0 & .95 \\
\hline
\end{tabular}

Note: $k=$ Number of Items in the subscales, $M=$ Mean, $S D=$ Standard Deviation, $\alpha=$ Reliability Co-efficient.

Table 2: Pearson Product Moment Correlation Analysis between Study Variables ( $\mathrm{n}=$ 150)

\begin{tabular}{llll}
\hline Variables & $\mathbf{1}$ & $\mathbf{2}$ & $\mathbf{3}$ \\
\hline 1. Rumination & - & $.54^{*}$ & $.80^{*}$ \\
\hline 2. Neuroticism & - & - & $.42^{*}$ \\
\hline 3. Depression & - & - & - \\
\hline
\end{tabular}

*. Correlation is significant at the 0.05 level (2 tailed).

**. Correlation is significant at the 0.01 level (2-tailed).

Table 3: Hierarchal Regression Analysis Predicting Depression on Rumination and Neuroticism $(\mathbf{n}=\mathbf{1 5 0})$

\begin{tabular}{llc}
\hline & \multicolumn{2}{c}{ Depression } \\
\hline Predictors & \multicolumn{1}{c}{ Nurses } & \\
\hline Step 1 & $.1 \mathbf{R}^{*}$ & $\boldsymbol{\beta}$ \\
\hline Rumination & & $.80^{*}$ \\
\hline Step 2 & $.31^{*}$ & \\
\hline Neuroticism & & $.42^{*}$ \\
\hline Total $\mathrm{R}^{2}$ & & \\
\hline Note: $* \mathrm{p}<.05 .{ }^{* *} \mathrm{p}<.01 ., * * * \mathrm{p}<.001$. & & \\
\hline
\end{tabular}

Dependent Variable: Depression

Predictors in the Models: Rumination, Neuroticism.

Table 4: Standardized Estimates of Direct Effects of the Pathway Analysis

\begin{tabular}{lllll}
\hline Predictors & Rumination & \multicolumn{3}{c}{ Depression } \\
\hline & $\beta$ & $S E$ & $\beta$ & $S E$ \\
\hline Neuroticism & -.10 & .11 & -.10 & .12 \\
\hline Rumination & & & $.47^{*}$ & .08 \\
\hline$R^{2}$ & & & \\
\hline
\end{tabular}


$* p<.05, * * p<.01, * * * p<.001$ Note: $\beta=$ Standardized coefficient, $\mathrm{SE}=$ Standardized error

Table 5: Standardized Estimates of Indirect Effect through Rumination

\begin{tabular}{lll}
\hline Predictors & Depression & \\
\hline & $\beta$ & SE \\
\hline Neuroticism & .04 & .10 \\
\hline
\end{tabular}

$*_{p}<.05, * * p<.01, * * * p<.001$ Note: $\beta=$ Standardized coefficient, $\mathrm{SE}=$ Standardized error

\section{Discussion}

The current research aimed to study the mediating role of rumination between neuroticism and depression among the married and unmarried nurses of Multan. The current study was also conducted to teach and provide insight to clinical settings on the disadvantages of depression. The outcomes of the current study comprise studied experience of nurses with a Pakistani community background, their rumination and neuroticism. The literature review provided the investigator's indication to assist and support the hypothesis of the present research.

The research was conducted to explore the effect of personality traits assessed early in their syllabus on stress responses and levels of depression and anxiety signs as junior physicians' subsequent graduation. Findings showed Neuroticism and reality weakness was related to high levels of perceived job stress (neuroticism $r=.19$, reality weakness $r=.17$ ) likewise higher levels of anxiety signs (neuroticism $r=.23$, reality weakness $r=.33$ ) and symptoms of depression (neuroticism $r=.21$, reality weakness $r=.36$ ) during the internship. Neuroticism indirectly predicted stress responses and levels of depression and anxiety signs. These relations were mediated through perceived job stress, whereas reality weakness predicted this psychological health assessment directly. Extroversion, on the other hand, protected against signs of depression $(\mathrm{r}=-.20)$. Moreover, females described higher levels of job stress than males $($ difference $=7.52)($ Gramstad et al. 2013).

A study was conducted by (Uliaszek et al. 2010) to assess the role of neuroticism and extraversion in the stress anxiety and stress depression relations among adults. Results revealed depression and social phobia were related with interpersonal CLS (IP-CLS), with neuroticism partially accounting for these relations. Depression was also related to noninterpersonal CLS (NI-CLS), but only in females. This research provides preliminary evidence for the significance of personality variables in explaining shared relations between stress and depression. Another research was conducted to study the association between positive affect, negative affect, rumination and depression, and to explore the mediating role of rumination between positive and negative affect and depression. Outcomes revealed that positive effects showed a significant negative association with rumination and depression. Likewise, there was a significant positive relationship between negative affect and rumination and depression. Furthermore, findings revealed that rumination partially mediated the association of positive and negative affect with depression (Ahadi et al. 2018).

A study was conducted to measure ruminative thoughts and their relation to depression and anxiety among nurses. Findings revealed that the relationship between rumination and depression was .33 , between rumination and anxiety was .32, and between depression and anxiety was .56 . The partial correlation between rumination and depression (controlling for 
anxiety level) was .20, and the partial correlation between rumination and anxiety (controlling for depression level) was .17. The result that rumination is not unique to depression but is also related to the specific negative effect of anxiety alone might also recommend new treatments of these two prevalent illnesses (Harrington et al. 1990).

Another research was conducted by (Piraman et al. 2016) to study the association between rumination and co-rumination among students. Findings showed that rumination $(\mathrm{P}=0.09)$ and co. rumination $(\mathrm{P}=0.09)$ had a significant and positive association with a total score of depression. Furthermore, the correlation of all three rumination subscales and all subscales of co-rumination except for the subscale in which "the person in question encourages his friends to discuss problems" had a positive and significant relationship with the total score of depression $(\mathrm{P}=0.01)$. On the other hand, Findings showed that the projection of students with depression in the case of rumination was significant $(\mathrm{P}=0.01)$.

\section{Implications}

The researchers found numerous implications when conducting the current study. The Researches came across a significant quantity of literature about the association between rumination, neuroticism and depression among nurses. Presently, only a short number of studies have been completed in Pakistani society. Though, the researcher hopes that the data collected from the current research will encourage others to learn more about the Pakistani society and population as well as encourage more researches with this population. On the early level, it would beneficial for experts to be up-to-date regarding the study variables and fulfill the previous research gap, particularly with the Pakistani community.

\section{Conclusion}

The main aim of this research is to dig out the mediating role of rumination between neuroticism and depression among the married and unmarried nurses of Multan. It is found that rumination partially mediates between neuroticism and depression meaning that neuroticism has a direct and indirect relationship with depression. It is concluded that depression may increase due to neuroticism as well as with the mediating effect of rumination in female nurses. This study will have significant theoretical and clinical implications, and could intensely improve our present knowledge about the transdiagnostic role that rumination, neuroticism and depression show an important role in psychopathology

\section{References}

Allan, C., \& Ebmeier, K. (2013). Review of treatment for late-life depression. Advances in Psychiatric Treatment, 19(4), 302-309.

American Psychiatric Association, \& American Psychiatric Association. (2013). Diagnostic and statistical manual of mental disorders: DSM-5. Arlington, VA.

Ahadi, B., Lotfi, M., \& Moradi, F. (2018). Relationship between positive and negative affect and depression: the mediating role of rumination. Practice in Clinical Psychology, 6(3), 191-196.

Broderick, P. C., \& Korteland, C. (2004). A Prospective Study of Rumination and Depression in Early Adolescence. Clinical Child Psychology and Psychiatry, 9(3), 383-394.

Bienvenu, O. J., \& Stein, M. B. (2003). Personality and anxiety disorders: a review. Journal of Personality disorders, 17(2: Special issue), 139-151. 
Clark, L. A., Watson, D., \&Mineka, S. (1994). Temperament, personality, and the mood and anxiety disorders. Journal of abnormal psychology, 103(1), 103.

Fang, L., Marchetti, I., Hoorelbeke, K., \& Koster, E. H. (2019). Do daily dynamics in rumination and affect predict depressive symptoms and trait rumination? An experience sampling study. Journal of behavior therapy and experimental psychiatry, 63, 66-72.

Gramstad, T. O., Gjestad, R., \& Haver, B. (2013). Personality traits predict job stress, depression and anxiety among junior physicians. BMC medical education, 13(1), 1-9.

Fanous, A., Gardner, C. O., Prescott, C. A., Cancro, R., \& Kendler, K. S. (2002). Neuroticism, major depression and gender: a population-based twin study. Psychological medicine, 32(4), 719-728.

Bagby, R. M., Ryder, A. G., Schuller, D. R., \& Marshall, M. B. (2004). The Hamilton Depression Rating Scale: has the gold standard become a lead weight?. American Journal of Psychiatry, 161(12), 2163-2177.

Harrington RC, Fudge H, Rutter M, Pickles A, Hill J. (1990). Adult outcomes of childhood and adolescent depression: I. Psychiatric status. Archives of General Psychiatry. 1990; 47:465-473.

Ibrahim, A. K., Kelly, S. J., Adams, C. E., \& Glazebrook, C. (2013). A systematic review of studies of depression prevalence in university students. Journal of psychiatric research, 47(3), 391-400.

Jacob, Y., Morris, L. S., Huang, K. H., Schneider, M., Rutter, S., Verma, G., \& Balchandani, P. (2020). Neural correlates of rumination in major depressive disorder: A brain network analysis. NeuroImage: Clinical, 25, 102142.

Klein, D. N., Durbin, C. E., \&Shankman, S. A. (2009). Personality and mood disorders. In I. H. Gotlib\& C. L. Hammen (Eds.), Handbook of depression (pp. 93-112). The Guilford Press.

Le Moult, J., Humphreys, K. L., King, L. S., Colich, N. L., Price, A. N., Ordaz, S. J., \& Gotlib, I. H. (2019). Associations among early life stress, rumination, symptoms of psychopathology, and sex in youth in the early stages of puberty: a moderated mediation analysis. Journal of abnormal child psychology, 47(2), 199-207.

Li, Y. I., Starr, L. R., \& Hershenberg, R. (2017). Responses to positive affect in daily life: Positive rumination and dampening moderate the association between daily events and depressive symptoms. Journal of Psychopathology and Behavioral Assessment, $39(3), 412-425$.

Li, J. J., Hilton, E. C., Lu, Q., Hong, J., Greenberg, J. S., \& Mailick, M. R. (2019). Validating psychosocial pathways of risk between neuroticism and late life depression using a polygenic score approach. Journal of abnormal psychology, 128(3), 200.

Muris, P., de Jong, P. J., \&Engelen, S. (2004). Relationships between neuroticism, attentional control, and anxiety disorders symptoms in non-clinical children. Personality and Individual Differences, 37(4), 789-797.

Murray, L. E., \& O'Neill, L. (2019). Neuroticism and extraversion mediate the relationship between having a sibling with developmental disabilities and anxiety and depression symptoms. Journal of affective disorders, 243, 232-240.

Meng, W., Adams, M. J., Reel, P., Rajendrakumar, A., Huang, Y., Deary, I. J., ...\& Smith, B. H. (2020). Genetic correlations between pain phenotypes and depression and neuroticism. European Journal of Human Genetics, 28(3), 358-366.

Murray, C. J., \& Lopez, A. D. (1997). Global mortality, disability, and the contribution of risk factors: Global Burden of Disease Study. The lancet, 349(9063), 1436-1442.

McCrae, R. R., \& John, O. P. (1992). An introduction to the five-factor model and its applications. Journal of Personality, 60(2), 175-215. 
Nutt, D., Demyttenaere, K., Janka, Z., Aarre, T., Bourin, M., Canonico, P. L., ... \& Stahl, S. (2007). The other face of depression, reduced positive affect: the role of catecholamines in causation and cure. Journal of Psychopharmacology, 21(5), 461471.

Nolen-Hoeksema, S. (1991). Responses to depression and their effects on the duration of depressive episodes. Journal of abnormal psychology, 100(4), 569.

Nolen-Hoeksema, S., Wisco, B. E., \&Lyubomirsky, S. (2008). Rethinking rumination. Perspectives on psychological science, 3(5), 400-424.

Neuendorf, R., Harding, A., Stello, N., Hanes, D., \& Wahbeh, H. (2016). Depression and anxiety in patients with inflammatory bowel disease: a systematic review. Journal of psychosomatic research, $87,70-80$.

Ormel, J., \&Wohlfarth, T. (1991). How neuroticism, long-term difficulties, and life situation change influence psychological distress: A longitudinal model. Journal of Personality and Social Psychology, 60(5), 744-755.

Ormel, J., Oldehinkel, A. J., \& Vollebergh, W. (2004). Vulnerability before, during, and after a major depressive episode: a 3-wave population-based study. Archives of general psychiatry, 61(10), 990-996.

Piraman, M. J., Tavakoli, M., \& Torkan, H. (2016). Rumination and co-rumination: Factors predicting depression. International Journal of Educational and Psychological Researches, 2(2), 99.

Sørengaard, T. A., Karlsen, H. R., Langvik, E., Pallesen, S., Bjorvatn, B., Waage, S., ... \& Saksvik-Lehouillier, I. (2019). Insomnia as a partial mediator of the relationship between personality and future symptoms of anxiety and depression among nurses. Frontiers in psychology, 10, 901.

Sokero, T. P., Melartin, T. K., Rytsälä, H. J., Leskelä, U. S., \& Isometsä, E. T. (2003). Suicidal ideation and attempts among psychiatric patients with major depressive disorder. The Journal of clinical psychiatry, 64(9), 0-0.

Smith, J. M., Alloy, L. B., \& Abramson, L. Y. (2006). Cognitive vulnerability to depression, rumination, hopelessness, and suicidal ideation: Multiple pathways to self-injurious thinking. Suicide and Life-threatening behavior, 36(4), 443-454.

Thanoi, W., Phancharoenworakul, K., Thompson, E. A., Panitrat, R., \&Nityasuddhi, D. (2010). Thai adolescent suicide risk behaviors: testing a model of negative life events, rumination, emotional distress, resilience and social support. Pacific Rim International Journal of Nursing Research, 14(3), 187-202.

Treynor, W., Gonzalez, R., \& Nolen-Hoeksema, S. (2003). Rumination reconsidered: A psychometric analysis. Cognitive therapy and research, 27(3), 247-259.

Uliaszek, A. A., Zinbarg, R. E., Mineka, S., Craske, M. G., Sutton, J. M., Griffith, J. W., ... \& Hammen, C. (2010). The role of neuroticism and extraversion in the stress-anxiety and stress-depression relationships. Anxiety, Stress, \& Coping, 23(4), 363-381.

Vergara-Lopez, C., Lopez-Vergara, H. I., \& Roberts, J. E. (2016). Testing a "content meets process" model of depression vulnerability and rumination: Exploring the moderating role of set-shifting deficits. Journal of Behavior Therapy and Experimental Psychiatry, 50, 201-208.

World Health Organization. (2008). The global burden of disease: 2004 update. World Health Organization.

Wang, K., Wei, D., Yang, J., Xie, P., Hao, X., \&Qiu, J. (2015). Individual differences in rumination in healthy and depressive samples: association with brain structure, functional connectivity and depression. Psychological medicine, 45(14), 2999-3008. 
Wells, K. B., Stewart, A., Hays, R. D., Burnam, M. A., Rogers, W., Daniels, M., ... \& Ware, J. (1989). The functioning and well-being of depressed patients: results from the Medical Outcomes Study. Jama, 262(7), 914-919.

Young, C. C., \& Dietrich, M. S. (2015). Stressful life events, worry, and rumination predict depressive and anxiety symptoms in young adolescents. Journal of Child and Adolescent Psychiatric Nursing, 28(1), 35-42.

Yousefi, Z. (2008). Rumination: beginning and continuous of depression. International Journal of Behavioral Sciences, 2(1), 67-73. 